\title{
LA DIGNIDAD COMO PRINCIPIO FUNDAMENTAL DE LA CONDICIÓN DE CIUDADANÍA DE LAS PERSONAS CON DISCAPACIDAD INTELECTUAL
}

\author{
Teresa Amezcua Aguilar \\ Marta Garcia Domingo \\ Virginia Fuentes Gutiérrez \\ Universidad de Jaén (España)
}

\begin{abstract}
Resumen. La desigualdad de las personas con discapacidad intelectual persiste a pesar de los logros alcanzados en las últimas décadas. Partimos de la premisa de que dicha desigualdad se debe, en gran medida, a que el concepto de dignidad, objeto de una evolución continuada a lo largo de la historia, continúa siendo confuso. Es por ello que el presente trabajo se propone analizar el concepto de dignidad desde una perspectiva histórica, hasta situarse en el contexto actual. A través de una revisión bibliográfica sistemática y del análisis de entrevistas exploratorias realizadas a informantes claves, abordamos los diferentes análisis teóricos sobre el concepto de dignidad que han respaldado los modelos de atención a la discapacidad a lo largo del tiempo. Entre los principales resultados encontramos que, de una significación jerárquica y de rango social de la dignidad -propuesta por los teóricos clásicos-, se pasa a definirla como un valor interno que hace único al ser humano como ser racional, con capacidad de elección y autónomo. Concluimos del análisis que los cambios de perspectiva sobre la dignidad sirven para acotar los posibles peligros que pueden vulnerar al ser humano, pero siguen siendo insuficientes a la hora de extender los derechos a una esfera más amplia que la personal.
\end{abstract}

Palabras Clave: Dignidad, Justicia social, igualdad, diversidad funcional

\section{THE DIGNITY AS A FUNDAMENTAL PRINCIPLE OF CITIZENSHIP STATUS OF PEOPLE WITH INTELLECTUAL DISABILITIES}

\begin{abstract}
The inequality of people with intellectual disabilities persists in spite of the achievements made in the last decades. We start from the premise that such inequality is largely due to the fact that the concept of dignity, object of a continuous evolution throughout the history, continues being confused. Because of it, the present work aims to analyze the concept of dignity from a historical perspective, to situate itself in the current context. Through a systematic literature review and the exploratory interviews with expert in the topic, we address different theoretical analyzes of the concept of dignity, supported by the different models of attention to disability over time. Among the main results we find that, from a hierarchical meaning and social rank of dignity - proposed by the classical theorists -, it is defined as an internal value that makes human being unique as a rational being, with a choice and autonomy . We conclude from the analysis that changes in perspective on dignity serve to limit the possible dangers that may harm human beings, but they are still insufficient when extending rights to a wider area than personal.
\end{abstract}

Keyword: Dignity, Social Justice, equality, functional diversity 


\title{
DIGNIDADE COMO O PRINCÍPIO FUNDAMENTAL DA CIDADANIA ESTATUTO DAS PESSOAS COM DEFICIÊNCIA INTELECTUAL
}

\begin{abstract}
Resumo. La desigualdade de pessoas com deficiência intelectual persiste apesar dos ganhos obtidos nas últimas décadas. Partimos da premissa amplamente que essa desigualdade é que o conceito de dignidade, sujeitos a evolução contínua ao longo da história, permanece obscuro. É por isso que este artigo é analisar o conceito de dignidade de uma perspectiva histórica, situando-se no contexto atual. Através de uma revisão sistemática da literatura e análise de entrevistas exploratórias com informantes-chave, abordamos a análise teórica diferente do conceito de dignidade que têm apoiado modelos de atenção deficiência ao longo do tempo. Os principais resultados são que, em um significado e gama dignidade -Proposta hierárquica social por teóricos Classics-, é passado para defini-la como um valor interno que é único sobre o ser humano, como um ser racional, capaz de escolha e autónoma. Análise concluiu que as mudanças em perspectiva sobre a dignidade servir para reduzir os possíveis perigos que possam violar o ser humano, mas ainda insuficiente quando se trata de alargar os direitos a uma mais ampla do que a esfera pessoal.
\end{abstract}

Palavras-chave: Dignidade, justiça social, igualdade, diversidade funcional

\section{Introducción}

La consideración de la universalidad de la dignidad humana hace necesaria la extensión de la Justicia social a toda la ciudadanía, premisa que choca con una realidad en la que la desigualdad es un hecho que persiste porque en nuestras sociedades se considera que la "diferencia" es un problema individual y ajeno. En el caso de las personas con discapacidad intelectual, éstas se encuentran, aún en pleno siglo XXI, en un espacio de singular vulnerabilidad en el que las medidas compensatorias que el Estado del bienestar proporciona son manifiestamente insuficientes para erradicar las situaciones de desigualdad social. Para la consecución de la igualdad de estas personas se hace necesaria una revisión de la conceptualización de la dignidad humana como pilar del ejercicio pleno de la ciudadanía. No obstante, son escasos los estudios que ponen el foco en los derechos de este colectivo y en los elementos que los fundamentan. Autores de referencia señalan que el reconocimiento de la dignidad de las personas con discapacidad intelectual pasa por un planteamiento de la Justica Social basado en la aceptación de la diversidad funcional (en adelante DF) (Sen, 2000; Nussbaum, 2006) y más concretamente de las personas con diversidad funcional intelectual (en adelante DFI) y de la diferencia (Fraser, 2008) de las personas dentro de la sociedad.

El reconocimiento de su dignidad como ciudadanos y ciudadanas se convierte en vehículo para la consecución del derecho al "respeto por las libertades y por la autonomía individual" (Sen, 2000), proporcionando garantías y protección ejemplificados por los derechos y libertades negativas, pero sin dar el paso definitivo de promoción de la dignidad, el que se concreta en el establecimiento de unas condiciones sociales y políticas que otorguen a la ciudadanía el reconocimiento de los derechos y libertades positivas: acceso a los bienes y servicios que han de ser organizados, coordinados y prestados por la sociedad y que procuran el desarrollo de la ciudadanía en condiciones de igualdad y libertad (Marshall y Bottomore,1998). Aunque teóricamente se reconoce su condición de ciudadanos y ciudadanas, para que el ejercicio de ciudadanía sea efectivo, se requiere de la puesta en marcha de políticas públicas 
garantistas, del reconocimiento social a su diferencia y de la contribución que ésta hace a la construcción de una sociedad más plural, diversa y rica.

En este artículo revisamos las diferentes perspectivas desde las que se ha contemplado la dignidad de las personas con DF en general y DFI en particular a lo largo de la historia, los planteamientos ideológicos y políticos que las han respaldado y los consecuentes modelos de atención a los que han dado lugar. El propósito de este trabajo es analizar las propuestas realizadas en este sentido por diferentes autores, poniéndolas en relación con los discursos expresados por informantes clave en el ámbito de la discapacidad intelectual. Con ello pretendemos ampliar el espectro teórico sobre la noción del principio de dignidad aplicado a este colectivo, insuficientemente desarrollada desde el ámbito de lo social. Este artículo pretende cubrir ese vacío de investigaciones concretas que reaviven el debate del reconocimiento de derechos de las personas con diversidad funcional intelectual.

\section{Método}

Nos aproximamos al objeto de estudio a través del empleo de una metodología cualitativa, basada en dos fases de investigación: $1^{\circ}$ ) meta-análisis de la conceptualización y reconocimiento de la dignidad de las personas con diversidad funcional en general, e intelectual en particular; y $2^{\circ}$ ) entrevistas semi-estructuradas a 6 informantes clave en la materia. En la primera fase se realizó una exploración bibliográfica y documental de fuentes clave a través de la revisión sistemática en bases de datos afines, a partir de los cuales abordamos los diferentes análisis teóricos sobre el concepto de dignidad que han respaldado los modelos de atención a la discapacidad a lo largo del tiempo. Elegimos aquellas bases de datos frecuentemente utilizadas en el ámbito de las Ciencias Sociales, como son: Latindex, Scopus, Dialnet, Redib, Miar, Erihplus, Doaj, Isoc y Google Académico. Para la búsqueda de fuentes realizamos una primera selección a partir de la exploración de textos clásicos en relación al tema y, posteriormente, realizamos una búsqueda avanzada que permite refinar la búsqueda mediante la combinación de distintos campos de información en el Directorio, Catálogo y Revistas en Línea. Para ello introdujimos descriptores de búsqueda avanzada afines al objeto de estudio tales como: "dignidad", "justicia social", "autodeterminación", "discapacidad", "igualdad" y "diversidad funcional". En la segunda fase realizamos una investigación exploratoria cualitativa a partir de entrevistas a 6 informantes clave en el ámbito de la discapacidad intelectual. La interpretación analítica de las mismas se llevó a cabo mediante la combinación del proceso de análisis de los discursos por parte de las investigadoras con el apoyo de herramienta informática ATLAS.ti en el proceso de categorización, estructuración y teorización.

Los principales hallazgos encontrados y la discusión y análisis de los mismos se plasman como resultados de la investigación. En concreto, estructuramos los hallazgos encontrados partiendo de lo más general para posteriormente pasar a focalizar la atención en lo particular. Para ello partimos del análisis de los modelos de atención a la discapacidad y su impacto en la concepción de la dignidad de las personas, para posteriormente adentrarnos en la autodeterminación del colectivo y examinar las principales fuentes de vulnerabilidad de su dignidad, relacionándolas con los discursos recogidos en el trabajo de campo. 


\section{Resultados y discusión}

Las formas en que la sociedad entiende la discapacidad determinan su mayor o menor inclusión social. A lo largo de la Historia ha habido tres modelos tradicionales de atención a la discapacidad que están estrechamente relacionados con la conceptualización que en cada época y sociedad se hace de la dignidad del ser humano.

En términos generales se entiende por dignidad el carácter de valía de algo o alguien. Pero este valor puede estar originado de dos formas: según una "versión fuerte" en la que la existencia de determinadas características proporcionan por sí mismos un valor inherente a quien las detenta, y una "versión débil", que arguye que la propia valía de algo o alguien le confiere razones para demandar una protección jurídica cuya realización dependerá, no obstante, de que la organización social en la que se encuentre reconozca la dignidad del valor a proteger y por tanto su dignidad (López de la Vieja, 2005). No obstante, las connotaciones de este concepto, aún hoy en día cargado de ambigüedad, han ido evolucionando a lo largo del tiempo, modificando no sólo la forma de entender la dignidad, sino también su categorización y los sujetos a los que es aplicable.

\section{Perspectiva evolutiva del concepto de dignidad de las personas con diversidad funcional desde los modelos de atención a la discapacidad}

\section{Modelo de prescindencia.}

En la época clásica por dignitas se hacía referencia a "cosa valiosa", que trasladado a la persona se entendía como condición de grandeza, rango, autoridad, características no aplicables a cualquier ser humano, lo que infiere un carácter restrictivo de la dignidad, circunscrita a su versión fuerte, que está reservada y relacionada con un determinado rango y papel de peso en el entramado social. La dignidad no es por tanto universal, no es inherente y requiere del cumplimiento de una serie de obligaciones relacionadas con el estatus social. A esta idea de dignidad responde el "modelo de prescindencia", que atribuye la discapacidad a causas religiosas (un castigo de los dioses ante una falta cometida). Deviene de una perspectiva política desde la que la discapacidad es vista como una carga para la sociedad por lo que, en las medidas adoptadas frente a ella, subyace el interés por su erradicación. Surgen de esta concepción dos submodelos: el modelo eugenésico, aplicado en la Antigüedad y el modelo de marginación imperante en la Edad Media (Toboso y Arnau, 2008). Estas dos formas de entender la discapacidad y se amparan en una idea de Justicia teocrática, que aplica la ley de selección natural basándose en los preceptos religiosos de cada época.

El modelo eugenésico se basa en la aplicación de las leyes biológicas orientadas al perfeccionamiento de la especie humana ${ }^{1}$.

La conceptualización cristiana de "dignidad", la entiende como el rango que obtiene el ser humano al ser creado a imagen y semejanza de Dios. No forma parte de su naturaleza, sino que le es concedida como una gracia. El pecado arrebata esta dignidad, le hace perder el rango social que le había sido dado. Desde la óptica cristiana la discapacidad es un problema individual del que la sociedad no es responsable, por lo que no tiene la obligación de asumir soluciones sociales. Surge así el modelo de

\footnotetext{
${ }^{1}$ Ejemplos de eugenesia son el abandono e infanticidio de los neonatos con discapacidad al considerar que su vida carece de valor social
} 
marginación cuya respuesta a las personas con discapacidad es individual y de atención asistencial (caridad y beneficencia), dando lugar a la exclusión social.

El surgimiento a finales del siglo XVIII de la Ilustración y las revoluciones liberales provocan un cambio definitivo del concepto dignidad. Los cambios sociales producidos por estos hechos convierten a todos los hombres ${ }^{2}$ en libres confiriéndoles una dignidad inherente a su condición humana. El sustrato político-filosófico de esta conceptualización la encontramos en la teoría del contrato social de Rousseau y la filosofía moral de Kant. A partir de ese momento los modelos de atención a la discapacidad se orientan hacia una concepción de la Justicia Social basado en la igualdad, por lo que la discapacidad no resta dignidad a la persona. Sin embargo, los nuevos modelos parten de enfoques diferentes sobre el origen de la discapacidad y su abordaje.

\section{Modelo médico o rehabilitador.}

Fue a principios del XX cuando esta nueva visión cristaliza. Los presupuestos de los que parte esta conceptualización de la discapacidad se bifurcan en dos vertientes: 1) Desde la perspectiva científica, el avance de la ciencia médica lleva a considerar que el origen de la discapacidad es psicofisiológica, por lo que ya no es vista como un castigo divino sino como un mal que aqueja a la persona y que puede ser tratado, curado e incluso prevenido, equiparándola a la enfermedad. 2) Desde la perspectiva social, las demandas sociales y las luchas obreras consiguen legislaciones proteccionistas de los derechos del trabajo y de la salud, como las primeras leyes de servicios sociales. Se analiza el papel de las personas con discapacidad en la sociedad, que ya no son consideradas como inservibles para la comunidad ya que, en el caso de poder ser "curadas" o rehabilitadas, pueden ser productivas y contribuir al desarrollo social.

Así, el modelo "médico o rehabilitador" justifica la discapacidad en causas médico científicas, percibiéndose como una dicotomía enfermedad/salud que lleva a buscar la "normalización" de las personas discapacitadas con el fin de que resulten socialmente productivas desde unos parámetros de estandarización funcional (Palacios y Romañach, 2008).

Por otro lado, siendo la rehabilitación un objetivo encomiable, presenta algunos dilemas de gran relevancia.

En primer lugar, el punto de partida de este modelo de atención se fundamenta en los principios del contractualismo, por los que cada individuo ha de aportar algo a la sociedad, según unos parámetros especificados desde la "ideología de la normalidad" y que se concretan en las capacidades de los individuos de realizar funciones de manera autónoma. Las características psicobiológicas de las personas con discapacidad se contraponen con los valores que fomenta la división social del trabajo en el modelo productivo capitalista. La discapacidad es invisibilizada al ser considerada un asunto médico individual, en el que la persona discapacitada se aleja de la "norma" socialmente aceptada y económicamente rentable, por lo que su segregación dentro del sistema productivo es resultado de un déficit individual (Guzmán, Toboso y Cabrero, 2010). Así, muchas de las políticas públicas relacionadas con la discapacidad "legitiman" la exclusión social de las personas con DFI al partir de la hipótesis de que ésta es un déficit médico individual. Al certificar la discapacidad se está dando una base

\footnotetext{
${ }^{2}$ Hay que resaltar que la restricción de la ciudadanía se ha circunscrito hasta hace bien poco a cumplir una serie de condicionantes que incluyen excepciones en función del género, la edad, la raza, el patrimonio, etc.
} 
para estigmatizar la discapacidad como una "anormalidad" de difícil cabida en el engranaje del mercado de trabajo (Oliver y Bochel, 1991). La Declaración Universal de derechos Humanos establece que los Estados deben desarrollar las políticas públicas necesarias para que los derechos humanos se hagan efectivos (ONU, 1948). No obstante, al dejar la implementación de las medidas oportunas al arbitrio de "la organización y los recursos de cada Estado", la Declaración deja abierta la puerta a la dejación de esta obligación por parte de los Gobiernos alegando falta de recursos.

En segundo lugar, este modelo ha fomentado la construcción de una identidad grupal estereotipada de las personas con DFI, que son identificadas por su discapacidad y no por su dignidad como personas. Un tercer dilema es la relación médico-paciente, que ubica a éste en una posición de inferioridad frente aquel y una preponderancia de los objetivos rehabilitación frente a los de inclusión social, que quedan en segundo plano (Palacios y Romañach, 2008). Finalmente, se produce una estigmatización de la persona discapacitada, quien es definida según unos estándares de normalidad, como revelan expresiones lingüísticas como minusválido, incapacitado, ínválido, subnormal o retrasado mental (Velarde, 2016). En este sentido, el discurso recogido en este estudio exploratorio identifica como una de las principales problemáticas el frecuente uso social de términos estereotipantes. Los participantes recalcan la importancia de realizar un avance terminológico que erradique las connotaciones negativas subyacentes en el vocabulario, tanto coloquial como científico, relativo a la diversidad funcional intelectual.

"Es necesario que dejemos de referirnos a ellos como discapacitados o minusválidos y que analicemos el daño que hacemos con esos términos. Y no hablo ya solo del daño que les podemos hacer a ellos, sino a la sociedad (...) a la percepción social" (E4).

Realizándose propuestas en pro de una reformulación del pensamiento colectivo a partir de la reconstrucción del lenguaje que favorezca el arraigo de una terminología no estigmatizante como vehículo de inclusión social.

\section{Modelo social.}

El paradigma de Justicia social que se plasma en la Declaración de Derechos Humanos de 1948 pone el foco en la obligación de los Estados de reconocer la igualdad de toda la ciudadanía y en la defensa de los derechos de las personas más vulnerables. La discapacidad pasa de considerarse un problema individual a una cuestión social, como ponen de manifiesto diferentes resoluciones de Naciones Unidas centradas en la protección de los derechos de las personas con discapacidad ${ }^{3}$. Este cambio de perspectiva origina un cambio de actitud ante la atención a la DI, surgiendo el "modelo social" de atención a la discapacidad.

El modelo social encuentra las causas de la discapacidad en las limitaciones de la sociedad para prestar los recursos y servicios que permitan cubrir las necesidades de las personas discapacitadas. De esta forma este modelo asume la discapacidad como un problema social, reconoce la "capacidad" de estas personas de realizar contribuciones sociales en la misma medida que cualquier otra, así como reivindica la autonomía de las

${ }^{3}$ Una muestra de ello es la "Declaración de los Derechos del Retrasado Mental" de 1971 o la "Declaración de los Derechos de los Impedidos" de 1975. 
personas con DFI para tomar decisiones sobre su propia vida, fomentando la eliminación de barreras que limiten la igualdad de oportunidades.

El sustrato filosófico del modelo social es el "enfoque de las capacidades" de Sen (2000) que surge en el contexto de la teoría del desarrollo humano. Para Sen el desarrollo no se circunscribe al crecimiento económico sustentado en el aumento de la producción de bienes, sino que tiene más que ver con lo que las personas pueden hacer realmente con los bienes que tienen a su alcance. Se conceptualiza la Justicia como distribución de bienes, entendiendo que una distribución desigual de los mismos produce desigualdades en las oportunidades de los individuos. Estas oportunidades favorecen la eliminación de desigualdades, barreras, dependencias, etc., dando lugar a nuevas oportunidades en la toma de decisiones y en la promoción de la autonomía. Así, la igualdad no es distribuir a todos por igual, ya que la igualdad entendida de esta manera (no equitativa), puede llevar a dar un trato desigual a quienes están en una posición desfavorable (Sen, 2000), sino que una sociedad es justa cuando los bienes están distribuidos de forma que las personas puedan hacer uso de ellos según sus capacidades para llevar a cabo la forma de vida que consideran valiosa.

Es en este punto donde los discursos de los participantes, conscientes de la diversidad funcional del ser humano, coinciden en manifestar como objetivo prioritario el respeto a su dignidad a la hora de elegir sus opciones de vida. Reclaman una igualdad de oportunidades que les permita aportar a la sociedad dependiendo de sus capacidades, desarrollando "funcionamientos" socialmente productivos, en el que el cómputo de resultados no sea medido desde un punto de vista económico, sino de Justicia social. Este modelo de atención traslada el punto de mira de lo individual a lo social. No es la persona con DFI la que debe ser rehabilitada (aunque sí recibir los apoyos necesarios), sino la sociedad la que debe rehacerse para adaptarse a la diversidad de capacidades y de funcionamientos, para empezar a beneficiarse de sus "talentos".

"La calidad de vida es consecuencia de que ha habido una autonomía para poder ejercer tu igualdad de oportunidades" (E6).

"Talentos que hay que descubrir y talentos que hay que...incorporar, herramientas y metodología de trabajo de manera que ese talento tenga un funcionamiento más o menos independiente y autónomo dentro de la propia persona" (E1).

"Bueno pues que la sociedad debería de ser un poquito más, más, menos acelerada, eso lo primero y lo segundo que podría ser un poquito más receptiva a lo diferente ¿no?, en este (...) bueno que son, no somos personas de segunda categoría, sino somos personas de primera categoría y que bueno, deberían de ser un poquito más... más cívicos, por decirlo de alguna manera. No ponernos más trabas de las que ya tenemos de por sí las personas con discapacidad; Y, bueno, en tema de política pues, que los políticos sean más... que se pongan más en el zapato de una persona con discapacidad" (E3).

En este sentido, el análisis de las entrevistas muestra que, pese a los avances ameritados, los informantes clave detectan que es la propia sociedad la que sigue obstaculizando la integración real de las personas con discapacidad intelectual. 
“(...) muchísimas dificultades de integración y conforme (...) conforme te vas haciendo más mayor, las dificultades son mayores, porque aunque nosotros intentamos que nuestros afiliados sean lo más autónomos posibles, la propia sociedad nos hace no ser autónomos. Es decir, no nos permiten ser autónomos" (E3).

Algunas críticas al enfoque de las capacidades señalan la necesidad de completarlo con una normativa que permita establecer cómo han de igualarse las capacidades como objetivo social (Nussbaum, 2007), ya que sólo se conseguirá una sociedad justa si se llega a un compromiso para lograr un conjunto de derechos para todas las personas. Este es otro de los problemas más reiterados en los discursos de los participantes, que expresan su preocupación por la falta de claridad y contundencia legislativa en esta cuestión. La solución a esta problemática pasa, según el estudio exploratorio realizado, por una clarificación de las capacidades y funcionamientos del ser humano con el objetivo de elaborar políticas públicas dirigidas a aumentarlas y promoverlas, y para poder llevar a cabo esto será necesaria una concepción universal de la dignidad de la persona y de la vida.

\section{Modelo de la diversidad funcional (DF).}

Desde el modelo de la DF se entiende que la dignidad tiene dos facetas: la dignidad intrínseca y la dignidad extrínseca (Palacios, 2008). La dignidad intrínseca tiene que ver con que todas las vidas tienen el mismo valor, la dignidad extrínseca está relacionada con la igualdad de derechos de todas las personas. Ninguna de estas acepciones del concepto dignidad se hace efectiva en el caso de las personas con DF.

Las políticas sociales actuales no han conseguido erradicar el trato discriminatorio hacia las personas con DF. Algunos autores califican como discriminatorias leyes como la del aborto, que estipula unos plazos diferentes al resto en los casos de DF del feto. Incluso la bioética plantea dilemas ante los avances tecnológicos aplicados a la genética que podrían dar pie en un futuro a la eugenesia. (Palacios y Romañach, 2008). Se argumenta que el problema está en la equiparación de los conceptos enfermedad y diversidad funcional, confusión que lleva a la búsqueda de la normalización funcional de las personas. Sin embargo, el modelo de DF incide en que la "normalidad" no existe, todos somos diferentes y tenemos derecho al reconocimiento a nuestra diferencia.

Así, frente a la teoría de la Justicia como distribución, individualizadora y consumista, que ha respaldado el modelo social de atención, surge una visión de Justicia como reconocimiento a la diferencia. La finalidad es la construcción de un mundo que acepte la diferencia y donde el relativismo moral o cultural no impida la plena integración de la ciudadanía (Fraser, 2008, p.83). Se propone un modelo en el que se amplíe la dicotomía capacidad/discapacidad por un concepto más amplio referido a la diversidad funcional como diversidad humana y en el que se sustituye como fundamentación teórica la dignidad por la capacidad. En contra de la propuesta de la Justicia como distribución que propugna la eliminación de las diferencias, la Justicia como reconocimiento propone poner en valor dichas diferencias, convirtiéndolas en seña de identidad del colectivo, buscando el respeto de la sociedad por la diversidad como elemento enriquecedor de la misma y reconociendo su dignidad por lo que son. 
Este elemento reconocedor de la diferencia también es destacado en las entrevistas, que dan luz al respecto de las oportunidades aparejadas a una sociedad que se construye en el conocimiento, la comprensión y la aceptación de las diferencia, dando cabida a las múltiples potencialidades asociadas a dicho reconocimiento:

"Por supuesto la diversidad está. Cada uno nacemos de nuestro padre y de nuestra madre (...) Estábamos hablando de que todas las personas somos diferentes, por cultura, por educación (...). A la altura que estamos de siglo, deberíamos estar todos preparados para poder trabajar, bueno, pues igual que tratas a unos que trates a otras personas" (E1).

"Talentos que hay que descubrir y talentos que hay que incorporar. Herramientas y metodología de trabajo de manera que ese talento tenga un funcionamiento más o menos independiente y autónomo dentro de la propia persona. (...) los talentos no se descubren y por eso las personas muchas veces no tenemos las oportunidades que llevamos innatas, dentro, ¿no? No tenemos las oportunidades de desarrollo” (E5).

\section{La dignidad como garante de atención a la diversidad funcional intelectual (DFI)}

La justa distribución de bienes y el reconocimiento a la diferencia no son suficientes para lograr una Justicia social plena para las personas con DF. La Justicia social requiere proporcionar vías que faciliten el acceso, en condiciones de equidad, a los sistemas y formas de participación social, a aquellas personas y colectivos que sufren una exclusión sistemática debido a su DF. Si no hay una participación libre y plena de la ciudadanía en los procesos de toma de decisiones no es posible una redistribución de bienes y posiciones sociales dentro de los parámetros de la Justicia social (Young, 2000). La estructura y organización social y el contexto institucional excluyen de los ámbitos de poder a los colectivos marcados por la diferencia, y de forma muy especial al de personas con DFI. De hecho, en este sentido, los participantes en este estudio mantienen la línea argumental de que la "igualdad de oportunidades" en el ámbito de la DFI se ha revelado más como una estrategia de marketing político que una verdadera ideología de inclusión social.

En definitiva, las estrategias emprendidas son deficitarias porque no cuentan con un método de trabajo sólido y constatado científicamente sobre el que sustentar una estructura de integración real y efectiva. Además, la falta de planificación real y la gestión ineficiente de los recursos escasos dan lugar a etapas inconstantes e inconsistentes, que dificultan una evolución coherente y constante.

"No es una cuestión de...de dinero ¡que también!, es una cuestión de etapas. Es una cuestión de planificación. No podemos echar un cubo de agua a un huertecito y no volver a echar más agua en meses. Si esa agua se hubiera gestionado en un periodo seguramente tendríamos garantizado cosechas durante unos meses. Pues yo creo que nunca hemos estado en la abundancia en la discapacidad" (E1)

"Estamos gestionando la supervivencia, donde muchos han abandonado. No hay estrategia, no hay plan de trabajo, no hay nada" (E5) 
Cabe destacar que, en este línea, los participantes denuncian un tratamiento diferencial desde la sociedad, en general, y las administraciones públicas, en particular, entre el colectivo de personas con DFI y otros colectivos de discapacidad, un agravio comparativo para el que no encuentran justificación y sobre el que, aseguran, pesan los prejuicios. En este sentido, y centrando la atención en la adaptación de los puestos de trabajo que favorezcan la integración de las personas con DFI en el mercado laboral, apuntan cuestiones como la siguiente:

"Pero cuando tú estás dentro de la empresa, haría falta, seguramente, pues resolver que la empresa se le facilite la entrada de una persona que de forma externa resuelva temas de fricciones entre los compañeros de trabajo, y estoy hablando en este caso de discapacidad intelectual básica. Yo he visto en empresas grandes como las personas con discapacidad intelectual que están trabajando se van solas, en el mismo grupo, a desayunar y no, al final, esos....esa integración en el mundo...laboral con tu propio grupo de trabajo, fuera no se produce. Entonces bueno, ahí habría que incorporar a lo mejor una serie de profesionales que permitieran el entendimiento" (E1).

En consecuencia, se plantea que, para la plena inclusión de este colectivo, es necesaria la redistribución de bienes no materiales que garanticen la igualdad de oportunidades para participar en distintos espacios públicos y acceder al poder. La solución está en una profundización en los procesos políticos de toma de decisiones que proporcionen igualdad real de oportunidades de participación a la ciudadanía con DFI.

"Que sean ellos los que propongan...que sean ellos los que los que analicen las nuevas situaciones" (E4).

"El problema de los colectivos sociales, es de los colectivos sociales. No tienen que esperar ni a que la administración, ni otras entidades de cualquier naturaleza les resuelvan el problema" (E6).

"Existe un modelo de inclusión sobre el tema de las personas vulnerables que deben de ser tenidas más en cuenta... También otra cosa importante, que se debería de tener en cuenta, es recuperar el sentido de pertenencia en esas personas: que puedan formar parte de algo, sentirse merecedoras de participar...y por supuesto también ser capaces de identificar las necesidades de forma colectiva y entonces, a partir de ahí, pues tomar las decisiones sobre la realidad que tienen esas personas" (E2).

"Normalmente, la gente participa a veces son los mismos y, muchas veces, la propia asociación es la que influye en esa participación. Porque aquí lo ideal sería que la gente participara en lo que realmente le gusta. (...) hay gente que tiene dificultad de acceso por problemas de pues de desplazamiento o problemas de tipo familiar para poder acompañar en estas actividades que se puedan celebrar (...) podemos contar que en una 
actividad participan casi siempre los mismos y en otra actividad. Lo que sí se intenta un poco es innovar para que la gente participe. Pero muchas veces influye mucho lo que comentaba, la dificultad que tienen al acceso a esa participación" (E5).

Una de nuestras informantes clave va más allá y aboga por un modelo de participación en el que las personas con DFI puedan realizar aportaciones no solo en el ámbito que directamente les incumbe (la DF), sino en cualquier otro que le sea de interés, bajo una concepción holística de la persona, sus intereses y capacidades.

“A nivel político se considera que yo solo puedo participar en los temas sociales de la discapacidad. No puedo participar en el ámbito de la energía, o el ámbito alimentario, cuando yo creo que hay todavía, pues eso, hay que entender a la persona con discapacidad precisamente porque es una persona que proyecta y se puede proyectar en muchas ámbitos y, sin embargo, aquí seguimos estando en este concepto de discapacidad y de las limitaciones que eso te produce" (E1).

Por otro lado, se apunta a la necesidad de construir un tejido social más cohesionado en el ámbito de la discapacidad, que deje de abogar por avances en pro de un solo tipo de discapacidad y luche de manera conjunta por el colectivo de DF en su conjunto.

"No tiene la misma fuerza la discapacidad junta que la discapacidad separada, entonces esa es otra fuerza (...) se consiguen bastantes más cosas cuando se está unido y cuando la discapacidad está unida y yo te digo desde el asociacionismo podemos hacer cambiar leyes" (E3).

"Si nosotros hiciéramos unas fotografías de los recursos que tenemos de personas con discapacidad que tenemos en la provincia de Jaén, nos quedaríamos impresionados, porque seguramente lo que antes era una red, como tú bien decías, pues ahora seguramente tenemos algo aquí, y el siguiente puesto estará allí. Donde no hay coherencia, donde no hay conexión, donde ni siquiera hay conexión en el propio municipio, que te podías apoyar aquí, te podías apoyar allí, y es....pero todo eso sin darnos cuenta, sin darnos cuenta. Todavía no hemos hecho esa terrible fotografía, hoy por hoy llamas a un teléfono y ya no existe, llamas, te interesas por un profesional ya no está, te interesas por una entidad, ya se ha ido..." (E1)

\section{La autodeterminación de las personas con diversidad funcional intelectual (DFI)}

La Clasificación Internacional del Funcionamiento, de la Discapacidad y de la Salud (CIF, 2015), se refiere a discapacidad como un término que engloba deficiencias, limitaciones de actividad y restricciones para la participación. Esta definición se centra en la interacción entre las características del organismo de las personas y la estructura de su propio medio social. En la misma línea, Schalock señala que "la discapacidad intelectual se caracteriza por limitaciones significativas tanto en el funcionamiento 
intelectual como en el comportamiento adaptativo, que se expresan en las habilidades conceptuales, sociales y de adaptación práctica" (Schalock, 2009, p.24). Se trata pues de un concepto complejo en el que entran en juego tanto las limitaciones del funcionamiento individual como las barreras que el contexto social dispone para su pleno desarrollo y que dan lugar a una situación de desventaja social.

La autodeterminación describe el grado de control que las personas tienen sobre todos los ámbitos de su vida, pudiendo tomar decisiones personales lo que implica un control absoluto sobre lo que incumbe a la persona. Para la población en general, la autodeterminación, se va logrando con el desarrollo de la persona hacia su vida adulta. Se inicia el control personal con la cesión por parte de los padres de decisiones sobre aspectos de la vida diaria considerados de menor importancia, como la hora de irse a la cama o el ocio (Smith, Morgan y Davidson, 2005). La asunción de potestad sobre aspectos de mayor importancia se va produciendo en relación a la edad y a la madurez psicológica: los adolescentes van tomando decisiones de mayor calado, como los relacionados con su itinerario educativo que afecta a su futuro profesional o el entorno social fuera del ámbito familiar. El aumento de la autodeterminación está relacionado con diferentes factores individuales: el sexo del sujeto, el grado de madurez emocional, social y cognitiva de los individuos y la percepción que sobre éstos aspectos tengan sus tutores (Peterson, 2013).

Las personas con DFI tienen un hándicap a la hora de lograr su autodeterminación. Las deficiencias cognitivas retrasan el momento de acceder al control de actividades de la vida diaria y mucho más en aspectos de mayor importancia en su desarrollo vital; incluso en no pocas ocasiones esta capacidad de elección se ve anulada a través de la incapacitación legal. Desde los modelos de atención clásicos la tendencia fue la institucionalización de las personas con DFI, lo que coartaba su capacidad de elección en cuanto a las actividades más básicas de la vida diaria (hora de acostarse, levantarse, qué comer, vestuario, etc.). La perspectiva ética planteada por el modelo social de atención condujo a la progresiva desinstitucionalización de las personas con DFI proponiendo tanto el mantenimiento en el entorno familiar como la promoción de otros modelos de convivencia a pequeña escala que facilitan desarrollar actividades de la vida diaria y estilos vida que se ajustan más a los del conjunto de la sociedad. Sin embargo, diferentes estudios ponen en evidencia que la convivencia en unidades institucionales de menor escala no produce siempre una mayor autodeterminación de las personas, sino que ésta depende de otros factores como el nivel de adaptación logrado por los residentes. En concreto, los estudios llevados a cabo por Parsons, McCarn y Reid (1993) y Sands y Kozleski (1994), al comparar unidades institucionales de convivencia de personas con y sin DFI evidenciaron que las personas con DFI tuvieron un nivel de acceso a oportunidades de acción claramente inferior al de los hogares de personas sin DFI. Otros estudios ponen en relación el grado de DFI con el nivel de oportunidades de acción para llevar a cabo actividades de la vida diaria, encontrándose que a mayor grado de DFI, menos capacidad de autodeterminación sobre decisiones que atañen al desempeño de este tipo de actividades (Smith et al., 2005). Estos resultados reflejan que las personas con DFI disfrutan de menos oportunidades de autodeterminación que la población en general.

En consonancia con estas investigaciones, una de las líneas argumentales más relevantes en este estudio incide en las dificultades de autodeterminación marcadas por una protección paternalista excesiva que comienza con el control parental y se traslada a las instituciones de guarda y tutela. 
"La propia sociedad nos hace no ser autónomos, es decir no nos permiten ser autónomos" (E3).

"Es una cuestión educacional. Lo primero, eh, todavía estamos rompiendo el, estereotipo de que cuando una persona con discapacidad intelectual hay que hacérselo todo. ¡No hay que hacérselo todo!;ni decidir por ellos! La semana pasada yo tuve una serie de ejemplos, que digo, madre mía, verdaderamente se te abren los ojos porque creíamos que habíamos avanzado en esto y sin embargo seguimos estando igual" (E1).

Del mismo modo, las políticas implantadas responden más a necesidades de atención de la dependencia que a la promoción de la autonomía real.

"Las políticas sociales están más diseñadas para lo que hemos hablado antes... Para el cuidado, para la protección, para la seguridad... Más que para el libre desenvolvimiento. Más que para el libre albedrío de las personas" (E6).

"Curiosamente, las ayudas que hacen que tú ganes en libertad... han sido las que han desaparecido" (E4).

Así, se reclama la puesta en marcha de medidas enfocadas a la sensibilización social en general, y a las partes implicadas, en particular, del derecho a decidir de las personas con DFI, que incluye el derecho a tomar decisiones controvertidas, fuera de la norma o lo convencional, e incluso el derecho a errar, del que disfrutamos la ciudadanía en general y que nos permite aprender y rectificar.

\section{La vulnerabilidad de la dignidad de la personas con DFI. El derecho al voto}

En determinadas situaciones la propia legislación que pretende garantizar los derechos de las personas con DFI vulnera su dignidad al cuestionar su autonomía moral. En muchas ocasiones se presupone falta de capacidad para tomar decisiones a personas con discapacidad intelectual teniendo como consecuencia la institucionalización de personas plenamente capacitadas para llevar una vida en igualdad de oportunidades si disfrutasen de los apoyos necesarios. La DFI puede también ser causa de incapacitación de la persona afectada, lo que determina su no capacitación para tomar ciertas decisiones por sí misma. En este sentido surgen múltiples dilemas morales sobre el respeto a la dignidad de las personas con diversidad funcional intelectual.

En razón al artículo 14 de la Constitución Española, las personas con DFI disfrutan de los mismos derechos que cualquier ciudadano, teniendo personalidad jurídica plena. La Ley General de derechos de las personas con discapacidad y de su inclusión social, define como principio rector el de Vida independiente: "la situación en la que la persona con discapacidad ejerce el poder de decisión sobre su propia existencia y participa activamente en la vida de su comunidad, conforme al derecho al libre 
desarrollo de la personalidad" ". Sin embargo también se previenen medidas judiciales en el caso de que la DI suponga unas limitaciones tales que deriven en un problema grave de la persona para autogobernarse: a) incapacitación total, b) incapacitación parcial, c) defensor judicial. Se estipulará cada una de ellas dependiendo de la situación psicológica, física o social de la persona para autogobernarse. El objetivo de estas medidas judiciales es la protección de los intereses y derechos del incapacitado a nivel personal, social y/o patrimonial. Hay que señalar que la incapacitación no restringe la titularidad de los derechos fundamentales pese a que sí que delimita la manera de ejercerlos. Es por ello que las sentencias de incapacitación deben ser exhaustivas, delimitando claramente los ámbitos en que se establece dicha incapacitación de acuerdo no sólo con el grado de discapacidad, sino también de su situación personal, familiar, de salud, económica, social, etc.

Las medidas de incapacitación tienen por finalidad la protección de las personas con DFI y, sin duda, aportan múltiples beneficios para a quienes tienen dificultades graves al realizar cierto tipo de actividades. A nuestro juicio, sin embargo, estas medidas han de limitarse a las situaciones estrictamente necesarias.

Diferentes estudios (Schalock, Hoffman y Keith, 1993; Schalock, 2009) han mostrado que la conducta adaptativa y el funcionamiento intelectual están íntimamente relacionados con la capacidad de tomar decisiones. Estos estudios evidencian que las oportunidades de autodeterminación son concluyentes en el desarrollo funcional de las personas, por lo que coartarlas puede provocar un menor desarrollo adaptativo de la persona con DFI. Desde nuestro punto de vista, para mantener intacta la dignidad de la persona con DFI, aunque medie una incapacitación legal del individuo, siempre ha de fomentarse el ejercicio del derecho de autodeterminación en la medida que sus capacidades lo permitan. Así, será necesario hacer partícipe a la persona de las posibles opciones de que dispone y proporcionarle los medios suficientes para tomar decisiones informadas, preguntándole por sus preferencias e intereses y teniendo en cuenta su opinión. Además, las personas con DFI sujetas a tutela o curatela pueden sentir una pérdida de control sobre su vida si la persona que la ejerce abusa de su poder no tomando en consideración sus preferencias y opiniones, aun cuando el tutor o tutora crea de buena fe estar haciendo lo mejor para la persona tutelada.

Otra circunstancia que se produce con frecuencia es que la incapacitación vulnere derechos fundamentales. Este es el caso del derecho al voto, sancionado en el artículo 23 de la CE sobre el derecho a participar en los asuntos públicos. A este respecto, se producen numerosas situaciones en las que se vulnera el derecho de las personas con DI legalmente incapacitadas, a pesar de que la CE exige a los poderes públicos que velen por el cumplimiento de los derechos constitucionales de las personas con discapacidad y de que la Convención de la ONU sobre los Derechos de las Personas con Discapacidad de 2006 estipula específicamente la obligación de los Estados de proteger los derechos políticos de las personas con discapacidad y su ejercicio en igualdad de condiciones. La Ley Orgánica de Régimen Electoral General estipula que carecen de derecho al sufragio "los declarados incapaces en virtud de sentencia judicial firme, siempre que la misma declare expresamente la incapacidad para el ejercicio del sufragio" (LOREG, 2011). Como señalamos anteriormente, la incapacitación debe ceñirse única y exclusivamente a los ámbitos en que la persona no pueda desarrollar determinadas actividades, ajustándose al máximo a sus circunstancias individuales. En

\footnotetext{
${ }^{4}$ Real Decreto Legislativo 1/2013, de 29 de noviembre, por el que se aprueba el Texto Refundido de la Ley General de derechos de las personas con discapacidad y de su inclusión social
} 
consonancia con esta línea argumental, los discursos de los participantes sostienen que el que una persona con DI esté bajo un proceso de incapacitación no significa que no tenga capacidad de discernimiento para elegir a sus representantes políticos si cuenta con los apoyos necesarios. Sin embargo, en la práctica, los jueces suelen utilizar formularios estándar para sentencias de incapacitación en los que incluyen por defecto la orden de comunicar dicha sentencia al censo electoral con la finalidad de que se retire a la persona incapacitada del censo, privándola así de su derecho a votar y ser electa. Lo que debería ser una excepción para casos muy concretos se convierte en una generalización amparada por la intención de proteger la posible manipulación del voto. Por ello, consideramos necesario que los procedimientos de incapacitación tengan en cuenta los factores que concurren en cada individuo, su capacidad de tomar decisiones fundamentadas en el ejercicio del derecho al voto y proporcionar las ayudas necesarias para que este derecho se haga de forma efectiva, eliminando las barreras cognitivas que entorpecen la comprensión del ejercicio del sufragio.

De acuerdo con esto, los participantes insisten en la importancia de que las personas con DFI accedan a las esferas de poder, resaltando que ello es sólo posible a través de una triple línea de actuación coordinada: 1) fomentando el interés de las personas con DFI por formar parte de las estructuras de poder; 2) articulando una red apropiada que dé cobertura a estos canales de participación política; 3) sensibilizando a la población de la necesidad de que este colectivo tenga una representación política de sus intereses.

\section{Conclusiones}

En este estudio realizamos un primer acercamiento a la realidad social de las personas con DFI desde el punto de vista de los teóricos y expertos en este ámbito, que nos lleva a señalar que no se ha dado aún el paso definitivo hacia la "promoción de la dignidad" de las personas con DFI, persistiendo las situaciones personales y colectivas de exclusión sistémica. Entre los principales resultados encontramos que, de una significación jerárquica y de rango social inicial de la dignidad, se pasa a concebirla posteriormente como un valor interno que hace único al ser humano como ser racional, con capacidad de elección y autónomo.

Para el reconocimiento social de la dignidad para las personas con DFI es necesario poner el énfasis no en la diferencia, sino en la naturalidad de la diversidad en la especie humana. Planteamos la consideración de la DFI como una propiedad inherente al ser humano que enlaza con la fundamentación de la biodiversidad natural. Sin embargo, se evidencia una distancia entre los discursos teóricos y los discursos de la experiencia de vida. Las personas con DFI continúan situándose en un escalón inferior en la consideración de su dignidad humana dentro del imaginario colectivo, como explicita el uso habitual de términos estereotipantes y peyorativos al referirlos. Nuestra propuesta en este sentido se dirige a la implementación de programas de divulgación y sensibilización que contrarresten las connotaciones negativas subyacentes en el léxico relativo a la DFI, tanto científico como coloquial, que avancen hacia una reformulación lingüística más centrada en la positividad de la diversidad que favorezca la inclusión social de estas personas.

Planteamos también que la noción ampliada de dignidad señala el camino hacia el que las políticas inclusivas debieran orientarse, atendiendo a una doble esfera: 1) la 
dignidad como parcela individual, reflejada en el respeto y la protección de derechos del ser humano; 2) la dimensión colectiva, formada por las relaciones sociales, los recursos existentes y la organización política y social, que estará guiada por la promoción de las capacidades y alternativas de la ciudadanía. Consideramos este último aspecto como una opción hacia la inclusión que permite además romper con los mitos sobre la DFI.

En definitiva, de lo planteado en este artículo, se vislumbra un panorama complejo en el que múltiples opiniones e intereses se enfrentan a la hora de considerar la autodeterminación de las personas con DFI. La dignidad confiere el derecho a tomar libremente decisiones sobre la propia vida. Una minoración de estas opciones de elección menoscaba claramente la dignidad de las personas con DFI. Nuestra propuesta en torno a esta discriminación paternalista desde los ámbitos familiar y social, legislativo e institucional converge hacia el desarrollo de medidas dirigidas a sensibilizar sobre el derecho a decidir de todas las personas, al margen de consideraciones biológicas o médicas. Este derecho a decidir debe de implicar también el derecho a tomar decisiones polémicas, fuera de los patrones tradicionales, inclusive el derecho a equivocarse, a cometer errores, porque cada fallo nos enseña algo que necesitamos aprender.

Finalmente, con este artículo perseguimos reavivar el debate en torno al reconocimiento efectivo de la dignidad de las personas con DFI como parte de la ciudadanía, no sólo en el espectro de la legalidad, que también, sino en el de la vida cotidiana. Para ello se han de construir nuevos patrones sociales de representación sustentados en el respeto a la diversidad que origine un cambio de calado en la sociedad.

\section{Referencias}

Fraser, N. (2008). La justicia social en la era de la política de identidad: redistribución, reconocimiento y participación. Revista de trabajo, 4(6), 83-99.

Gobierno de España. (2006). Ley, 39/2006, de 14 de diciembre, de Promoción de la Autonomía Personal y Atención a las personas en situación de dependencia. En BOE, 299, de 15 de diciembre de 2006, 44142-44156.

Gobierno de España. (2011, 28 de enero). Ley Orgánica 3/2011, de 28 de enero, del Régimen Electoral General. En BOE núm. 25, de 29 de enero de 2011, páginas 9504 a 9523.

Guzmán, F., Toboso, M. y Cabrero, J. R. (2010). Fundamentos éticos para la promoción de la autonomía: hacia una ética de la interdependencia. Alternativas: Cuadernos de Trabajo Social, (17), 45-62.

López de la Vieja, M. T. (2005). Dignidad, igualdad. La 'buena' política europea”. Ciudadanos de Europa. En M. T. López (coord.), Derechos fundamentales en la Unión Europea (pp 79-103). Madrid: Biblioteca Nueva.

Marshall, T. H., y Bottomore, T. B. (1998). Ciudadanía y clase social. Madrid: Alianza Editorial.

Nussbaum, M. C. (2006). Las fronteras de la justicia: consideraciones sobre la exclusión. Paidós. 
Oliver, M., y Bochel, H. M. (1991). The politics of disablement. International Journal of Rehabilitation Research, 14(2), 185. doi: https://doi.org/10.1097/00004356199106000-00012

ONU. (2008). Declaración Universal de los Derechos Humanos, United Nations. Art. 22

OMS (2001). Clasificacion internacional del funcionamiento, de la discapacidad y de la salud (CIF). Madrid: IMSERSO.

Palacios, A y Romañach, J. (2008) "El modelo de la diversidad: una nueva visión de la bioética desde la perspectiva de las personas con diversidad funcional (discapacidad)". En T Ausín. y R. Aramayo (eds.), Interdependencia. Del bienestar a la dignidad. Madrid: Plaza y Valdés.

Parsons, M. B., McCarn, J. E., y Reid, D. H. (1993). Evaluating and increasing mealrelated choices throughout a service setting for people with severe disabilities. Journal of the Association for Persons with Severe Handicaps, 18(4), 253-260. doi: https://doi.org/10.1177/154079699301800408

Peterson, C. C. (2013). Looking forward through the lifespan: developmental psychology. Pearson Higher Education AU.

Sands, D. J., y Kozleski, E. B. (1994). Quality of life differences between adults with and without disabilities. Education and Training in Mental Retardation and Developmental Disabilities, 90-101.

Schalock, R. L. (2009). La nueva definición de discapacidad intelectual, apoyos individuales y resultados personales. Siglo Cero, 40(1), 22-39.

Schalock, R. L., Hoffman, K., y Keith, K. D. (1993). Quality of life questionnaire manual. International diagnostic systems publishing Corporation. Worthington, $\mathrm{OH}$.

Sen, A. (2000). Nuevo examen de la desigualdad. Alianza Editorial. Madrid.

Smith, R., Morgan, M., y Davidson, J. (2005). Does the daily choice making of adults with intellectual disability meet the normalization principle? Journal of Intellectual and Developmental Disability, 30(4), 226-235. doi: https://doi.org/10.1080/13668250500349359

Toboso, M. y Arnau M. S. (2008). La discapacidad dentro del enfoque de capacidades y funcionamientos de Amartya Sen. Araucaria: Revista Iberoamericana de filosofía, política y humanidades, (20), 64-94.

Velarde, V. (2016). Los modelos de la discapacidad: un recorrido histórico. Revista Empresa y Humanismo, 15(1), 115-136.

Young, I. M. (2000). La justicia y la política de la diferencia (59). Valencia: Universitat de València.

Fecha de recepción: 09/10/2017

Fecha de revisión: 31/10/2017

Fecha de aceptación: 23/03/2018 